\title{
ANALYSIS OF THE VISTA LONGITUDINAL SIMULATION CAPABILITY FOR A CRUISE FLIGHT CONDITION
}

\author{
Ba T. Nguyen \\ Lynne T. Hamilton-Jones, Capt \\ David B. Leggett \\ Advanced Development Branch \\ Flight Control Division \\ Wright Laboratory \\ Wright-Patterson AFB, OH 45433
}

\section{ABSTRACT}

The Variable Stability In-Flight Simulator Test Aircraft (VISTA) is the next generation US in-flight simulator for high-performance aircraft currently being developed by the US Air Force. VISTA is an F-16D in which the cockpit environment, feel, and flying characteristics can be changed to match a wide array of aircraft. While VISTA can simulate a wide range of dynamics, its capabilities are subject to certain practical limitations due to aircraft mass and inertia, available control power, actuator bandwidth, computational delays, structural modes, etc.

The purpose of this analysis is to estimate the range of dynamic response characteristics that the VISTA can simulate for the longitudinal mode of an up-and-away flight condition. The analysis matched the angle-of-attack time response of a Low-Order Model (LOM) to a linear simulation of the VISTA aircraft for a range of feedback gain sets. In the analysis presented here, a frequencydomain matching technique with the equivalent time delay included in the optimization of the match was used. The effects of the feedback gain sets to the VISTA aircraft dynamics will be shown. The result of this analysis will be compared against the VISTA goal.

List of acromyms

$\begin{array}{ll}\text { ADPO } & \text { Advanced Developement Program Office } \\ G_{\alpha} & \text { Alpha Feedback Gain } \\ \mathrm{G}_{\dot{\alpha}} & \text { Alpha Dot Feedback Gain } \\ \alpha & \text { Angle of Attack } \\ \dot{\alpha} & \text { Angle of Attack Rate } \\ \tau_{e} & \text { Equivalent Time Delay } \\ \omega_{\boldsymbol{*}} & \text { Equivalent Frequency }\end{array}$

$\zeta$

HOM

LOM

$\mathbf{N}_{\mathbf{x}}$

G $\quad$ Normal Acceleration Gain

$\mathbf{q}$

$\mathbf{G}_{\mathrm{q}}$

$\lambda_{1}, \lambda_{2}$

TPS

VISTA

High Order Model

Lower Order Model

Pitch Rate

Pitch Rate Feedback Gain Function

Test Pilot School
Equivalent Damping Ratio

Normal Acceleration at CG

Negative Roots of Second Order Transfer

Variable Stability In-Flight Simulator Test Aircraft

\section{INTRODUCTION}

The ability for VISTA to simulate other aircraft is provided by the Variable Stability System (VSS). The VSS is a network of three Rolm Hawk computers which compute control commands to the F-16 actuators to simulate flight characteristics of the vehicle under consideration. This involves interfacing the VSS with the existing F-16 digital fly-by-wire control system. The VSS will use the response-feedback technique to match the motions of the aircraft being simulated. Gain scheduling will be incorporated to account for nonlinearities in the aerodynamics.

VISTA has four primary applications. The first is in-flight simulation for new aircraft development and pre-first flight flying qualities test. The second is training test pilots in the Air Force and Navy test pilot schools. The third is flight control research on flying qualities. The fourth is flight vehicle integration studies of combat maneuvering and avionics/flight control system integration. VISTA is being built by General Dynamics with the Calspan Corp responsible for the development of the VSS. 
The baseline VISTA simulation envelope is defined to be $80-90 \%$ of the F-16D envelope and less than Mach 0.9. This represents a significant increase over the existing US. in-flight simulators. Figure 1 shows the light envelopes of in-flight simulators.

\section{ANALYSIS APPROACH}

For this analysis, a non-real time, linear, six-degree-of-freedom, High-Order Model (HOM) simulation of the VISTA aircratt using Matrixx, control design and analysis program, was used to determine the frequency response characteristics for a range of feedback gain sets. For each gain set, a frequency-domain matching program, McFit, was used to obtain a LOM for each set of gains. The range of LOM response characteristics were plotted to graphically illustrate the VISTA simulation capability.

\subsection{High Order Model}

The HOM created with Matrixx consisted of linearized F-16 plant dynamics, fourth-order actuator models, and second-order Pade' approximation for $18 \mathrm{msec}$ system transport time delay (e-").

The $18 \mathrm{msec}$ system transport time delay was modelled for the Hawks computation. A block diagram of HOM is shown below. This model was validated with a check case from a nonlinear sixdegree-of-freedom simulation. Time histories of the nonlinear simulation (Calspan's HOM) check case and the corrsponding Advanced Development Program Office (ADPO) HOM response are shown in figure 2.

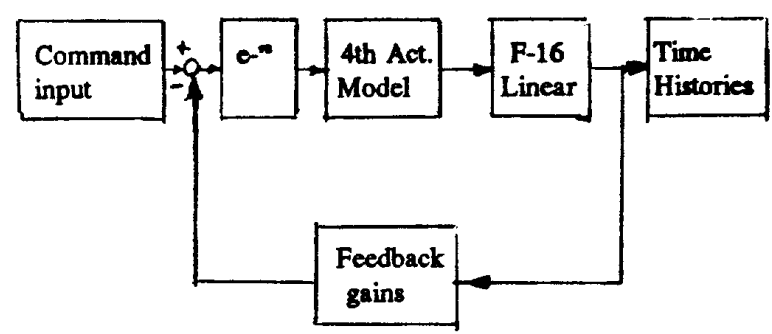

Linear High Order Model

For longitudinal dynamics, there are four feedback gains: angle of attack $\left(G_{\nsim}\right)$, angle of attack rate $\left(G_{9}\right)$, pitch rate $\left(G_{4}\right)$, and normal acceleration $\left(G_{z}\right)$. The dynamics of VISTA can be altered by adjusting the feedback gains. For this analysis the range of values used for each feedback gain was limited to those used by Calspan in their analysis. The $G_{z}$ was fixed at 0.0 . The $G_{\alpha}$ ranged from 0.0 to 6.0. The $G_{q}$ ranged from 0.0 to 0.6 . The $G_{a}$ ranged from 0.0 to 0.1 . The limits of these feedback gains were based on the Calspan experience. Different combinations of these feedback gains were set in the HOM on Matrixx. For combinations of these feedback gains, the transfer function of the delta elevator command to angle of attack response was determined.

\subsection{Lower Order Model}

Once the dynamic characteristics of the HOM were eatablished for a given set of feedback gains, the McFit program was used to find the LOM which most closely mitched the HOM transfer function. The form of the LOM used in this analysis is shown in the block dingram below. The LOM parameters were the equivalent time delay $\left(r_{0}\right)$, the equivalent damping ratio $\left(\zeta_{0}\right)$, the equivalent frequency $\left(\omega_{0}\right)$, and the equivalent transfer function gain $(k)$. Occasionally, the LOM denominator found by McFit consisted of two real roots instead of a complex pair. In such cases the denominator was dewcribed by $\left(s+\lambda_{1}\right)\left(s+\lambda_{2}\right)$.

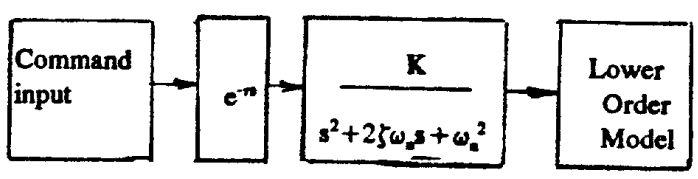

Linear Low Order Model

\subsection{Matching Technique}

The McFit program was developed by the McDonnell Aircraft Company to be used to determine equivalent lower order models of highly augmented aireraft. It is frequently used by the Flight Dynamics Diroctorate and the Acronautical Systems Division to determine compliance of such aircnt with the US military flying qualities specifications.

McFit will match, in the frequency domain, a high order transfer function or frequency response dath with in equivalent reduced order model. It uses a weighted least-square optimal match of a high order frequency response in amplitude and phase angle at up to fifty frequencies in a user-specified frequency range. The program adjusts the parameters of the reduced-order equivalent system in an iterative multivariable search until a cost function is minimized. The cost function used in McFit is:

$\omega$.

Cost $=(20 / n) \sum_{\omega_{1}}\left[\left(\text { gain }_{\text {HOM }}-\text { gain }_{\text {LOM }}\right)^{2}+.01745\left(\text { phase }_{\text {HOM }}-\text { phase }_{\text {LOM }}\right)^{2}\right.$

where: gain is in $\mathrm{dB}$ phase is in degrees

$\approx$ is the input frequency

$n$ is the number of discrete frequencics

For this analysis, the LOM was matched to the HOM at 30 frequencies in the frequency range from 0.1 to $15 \mathrm{rad} / \mathrm{sec}$. Guidance in the flying qualities standand, MIL-STD-1797, recommended a frequency range of interest from 0.1 to $10.0 \mathrm{rad} / \mathrm{sec}$ unless the equivalent short-period frequency was greater than 10.0 
$\mathrm{rad} / \mathrm{soc}$. In this analysis, there were several LOMs with equivalent short-period frequenciea greater than $10.0 \mathrm{rad} / \mathrm{sec}$. Therefore, 15 $\mathrm{rad} / \mathrm{sec}$ was used as the upper frequency range limit for all matches done in this analysis. By doing this, the cost function would be the same for all matches. All of the parameters of the LOM were freed. That is, the search routine could adjust all of the parameters, including $\tau_{0}$ in the optimization of the match.

\section{ANALYSIS RESULTS}

The analysis effort concentrated on determining the VISTA simulation boundary in the longitudinal axis for the following cruise flight condition:

$$
\begin{aligned}
& V_{1}=350 \text { kts (indicated air speed) } \\
& \text { Mach }=0.78 \\
& \text { Gear and Flap up } \\
& \text { Altitude }=20,000 \text { feet above sea level }
\end{aligned}
$$

\subsection{VISTA Short-Period Simulation Boundaries}

The predicted VISTA short-period simulation boundary is graphically depicted in figure 3 . This figure requires some explanations to assist interpretation. The scale across the bottom is $2 \zeta_{8} \omega_{2}$ or $\lambda_{1}+\lambda_{2}$. The scale on the right-hand is $\boldsymbol{\vartheta}^{2}$ or $\lambda_{1} \lambda_{2}$. The scale of the left-hand side is $\boldsymbol{\sigma}_{e}$ or square root of $\lambda_{1} \lambda_{2}$. The parabolic lines represent lines of constant equivalent damping ratio, $\zeta_{\text {. }}$ This form allows plotting of complex pairs and real pairs on the same plot. The thick lines indicate the boundaries of VISTA shortperiod simulation capabilities as a function of equivalent time delay. The bottom boundary is the $G_{\alpha}=0.0$ limit. The top boundary is the $G_{a}=6.0$ limit. The left most boundary is the $G_{q}=0.0$ limit. The boundary in the upper left comer is the $G_{9}=0.6$ limit. The $r_{e}=80 \mathrm{msec}$ line is the second line from the left. Any points on this line can be simulated with no less than $80 \mathrm{msec}$ of equivalent time delay. For this flight condition, the VISTA aircraft could not simulate dynamics with less than $70 \mathrm{msec}$ of equivalent time delay. The boundary on the far right is the $\tau_{0}=120 \mathrm{msec}$ line. Of course, the VISTA aircraft can simulate dynamics with higher time delay than $120 \mathrm{msec}$. However, the VISTA ADPO did not have enough points to describe the VISTA simulation boundary beyond this line.

The $\tau_{e}$ boundary lines indicate that it requires a higher equivalent time delay to simulate higher equivalent short-period frequencies or damping ratio. For example, looking at figure 3 it requires approximately $85 \mathrm{msec}$ of equivalent time delay to simulate an airplane with $\mho_{c}=5 \mathrm{rad} / \mathrm{sec}$ and $\zeta_{e}=.2$. It requires aproximately $94 \mathrm{msec}$ of equivalent time delay to simulate an aircraft with $\boldsymbol{\omega}_{\mathrm{e}}=$ $5 \mathrm{rad} / \mathrm{sec}$ and $\zeta_{e}=.6 \mathrm{rad} / \mathrm{sec}$. It requires approximately $114 \mathrm{msec}$ of equivalent time delay to simulate an aircraft with $\mho_{c}=10$ $\mathrm{rad} / \mathrm{sec}$ and $\zeta_{0}=0.6$. Using category $A$ flying qualities in the standard handbook, MIL-STD-1797, the $\tau_{e}, \omega_{e}, \zeta_{e}$ boundary lines also describe the VISTA simulation capabilities in terms of flying qualities in figure 4. Figure 5 shows the predicted VISTA shortperiod simulated boundary overlaid with the VISTA goal.

\subsection{Relationship Between Feedback Gains and LOM Dynamics}

Figures 6-9 show the relationships between the feedback gains and equivalent dynamics of the LOMs. Figure 6 show a short period frequency as a function of $G_{\text {and }}$ and. While holding the $G_{\varepsilon}$ and $G_{z}$ constant and increasing $G_{q}$, the frequency will increase respectively. Figure 7 shows a short period damping ratio as a function of the $G$. and $G_{\text {, with the }} G_{\text {: fixed at }} 0.0$. The damping ratio increased faster

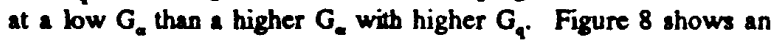
equivalent time delay as a function of the $G_{a}$ and $G_{a}$ with the $G_{8}$ fixed at 0.0 . The equivalent time delay increased faster at a low $G$. than a higher $G_{\text {. with higher }} G_{\text {v }}$.

\subsection{Time Integnal Error Model}

The analysis described in this paper measures the LOM match to the HOM by the cost function as described above. To comply with one of the VISTA statement of wort requirements, this analysis used the following equation for the time integral error.

$1 / \Delta \int_{t_{1}}^{t_{2}} \mid$ Xmodel - Xvista $\left.\right|_{d t} \leq .15 \quad 1 / \Delta \int_{t_{1}}^{t_{2}} \mid$ Xmodel $\left.\right|_{d t}$

Where: Xmodel is the model representing the HOM Xvista is the model representing the LOM $t_{1}$ is the beginning of the time interval of interest $t_{2}$ is the end of the time interval of interest $\Delta=t_{2}-t_{1}$

The block diagram below represents the above equation.

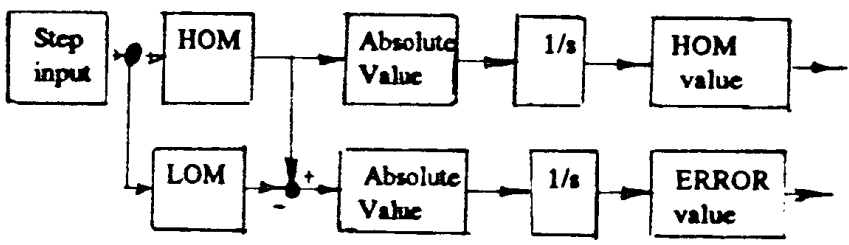

For this analysis, the Xmodel wzs the ADPO's linear model on Matrixx for each of the five gain sets. For each linear model, the corresponding Xvista was determined from McFit. A step input with a magnitude of one was used, and the time interval of interest was from 0 to 4 seconds. The following table contains the result of LOM matches for five selected gain sets: 


$$
\begin{aligned}
& \text { conf. } 5 \text { Time } \\
& \text { no. (rad/ delay } \\
& \text { sec) (msec) }
\end{aligned}
$$

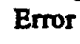

G. $\mathbf{G}_{\mathbf{q}} \mathbf{G}_{\text {- }}$

(a)

1

$\begin{array}{lllll}0.04 & 5.8 & 83 & 4.0 & 3.2\end{array}$

$\begin{array}{lll}2.0 & 0.1 & 0\end{array}$

2

$\begin{array}{lllll}0.15 & 6.3 & 88 & 5.6 & 2.2\end{array}$

$\begin{array}{lll}2.0 & 0.2 & 0\end{array}$

$\begin{array}{lllll}0.58 & 5.4 & 97 & 7.6 & 1.2\end{array}$

$\begin{array}{lll}1.0 & 0.3 & 0\end{array}$

$\begin{array}{lllllllll}0.31 & 10.31 & 109 & 16.9 & 2.2 & 3.0 & 0.5 & 0.5\end{array}$

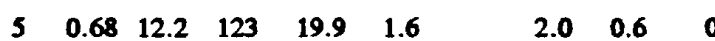

\section{OBSERVATIONS}

Based on this analysis, the VISTA simulation boundary was formed for the longitudinal axis. There are couple of thinga to remember:

4.1 The LOM match ignored the phugoid mode of the HOM.

4.2 Compare the LOM time history with the HOM time history respectively to check the model fidelity by using the time integral error criterion.

4.3 Sometimes, the McFit matching program did not match the HOM very well for the case where the HOM had overdamped characteristics. In other words, the HOM has two real roots instead of two complex roots. It can be matched better with the first order LOM instead of the second order LOM.

4.4 The analysis found that there was a slight difference in the dynamic response for the pitch rate HOM match compared to the alpha HOM match. Some of the pitch rate HOM matches did not pass the time integral error criterion of the statement of work. while the alpha HOM matches did. Both HOMs used the same feedback gains. The difference between the LOMs was the damping ratio. The plots of the pitch rate and the alpha HOM match are shown in figures $9 a$ and $9 \mathrm{~b}$ respectively.

\section{CONCLUSION}

The analysis presented in this paper indicates that the VISTA aircraft does not meet the goal for up-and-away short-period mode. VISTA - does not meet the requirement for having less than 70 msec of ivalcat time delay between the model and VISTA aircraft response for an aircraft with no time delay. This will have less affect in practice because all modern aircraft have time delay due to actuators and computations from hardware and software. It should be pointed out that the ganls for the VISTA simulation dynamics were based on the incoporation of high bandwith setuators. Instead, baseline VISTA will use the existing F-16 actuation with resulting docreasing performance capability. Athough not validated by the VISTA ADPO, Calspan's analysis demonstrates that the VISTA sireraft meets the goal for power approsch short-period modes. It also meets the goal for the hateraldirectional modes for both up-and-away and power approach flight conditions.

\section{References}

[1] Calspan TM No. 120, Analysis of the VISTA Longitudinal Simulation Capability for the Cruise Flight Condition,' 30 January 1990.

[2] M.V Dunbar, J.L Dargan, 'VISTA/F-16 Design Features,' AIAA Conference.

[3] General Dynamics, 'Class H-1 Modification Document for VISTA,' Preliminary Document, March, 1989.

[4] General Dynamics, 'Critical Design Review Viewgraphs,' VISTA CDR, October, 1989.

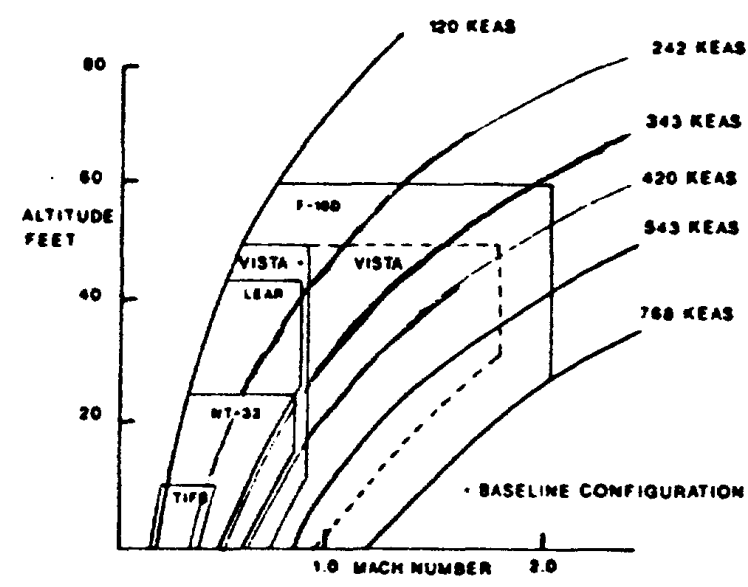

Fig. 1 U.S. Simulators Performance Envelopes 


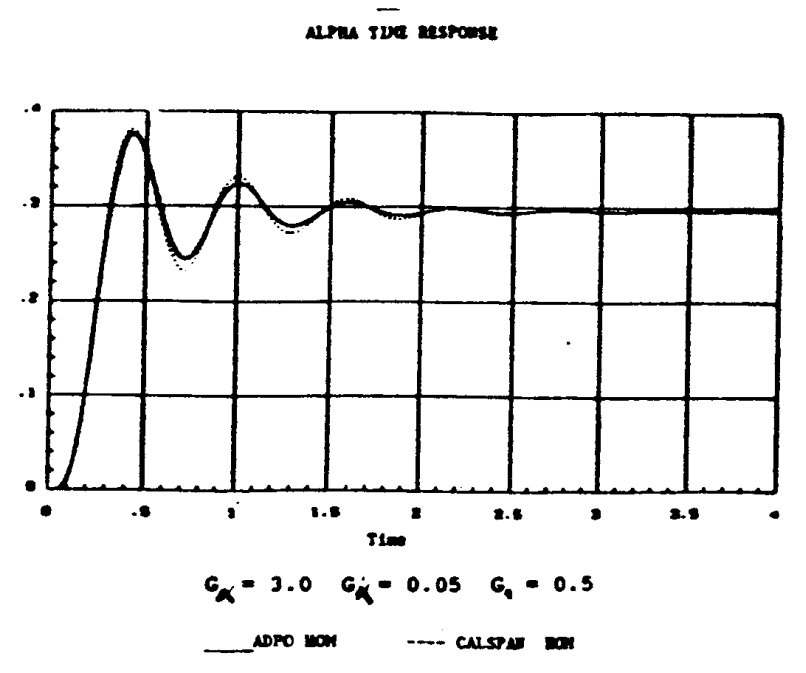

Fig. 2

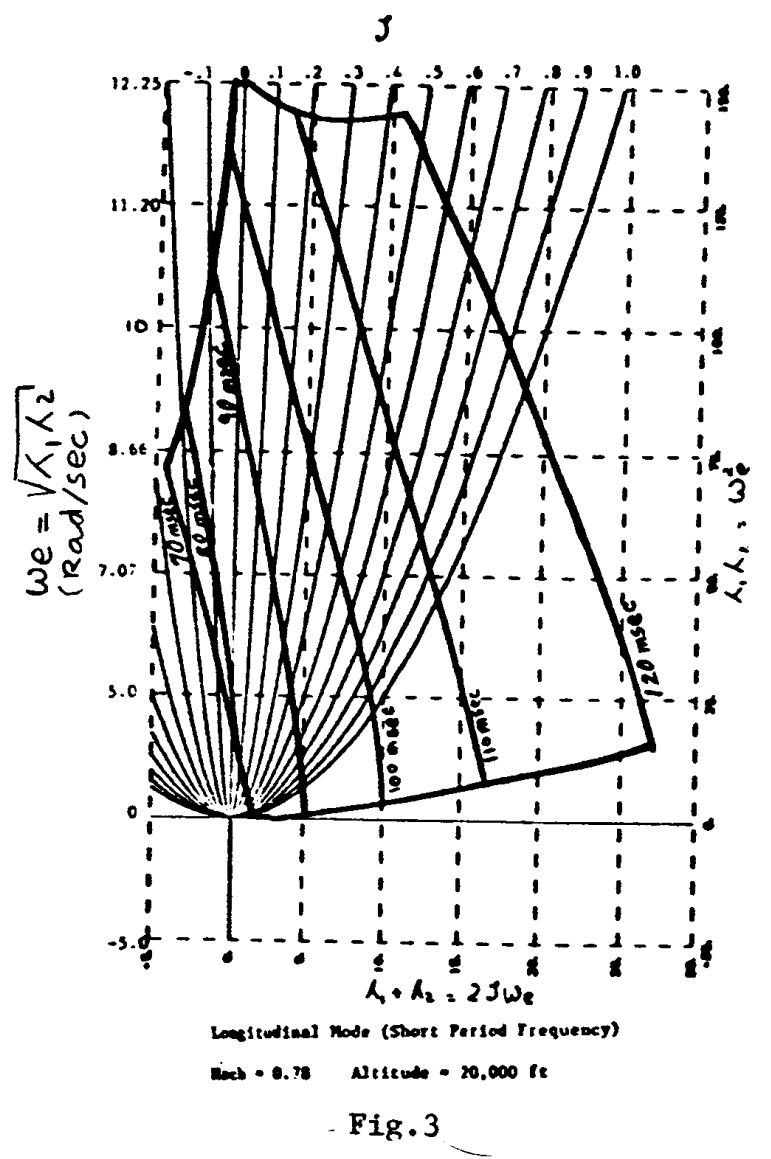

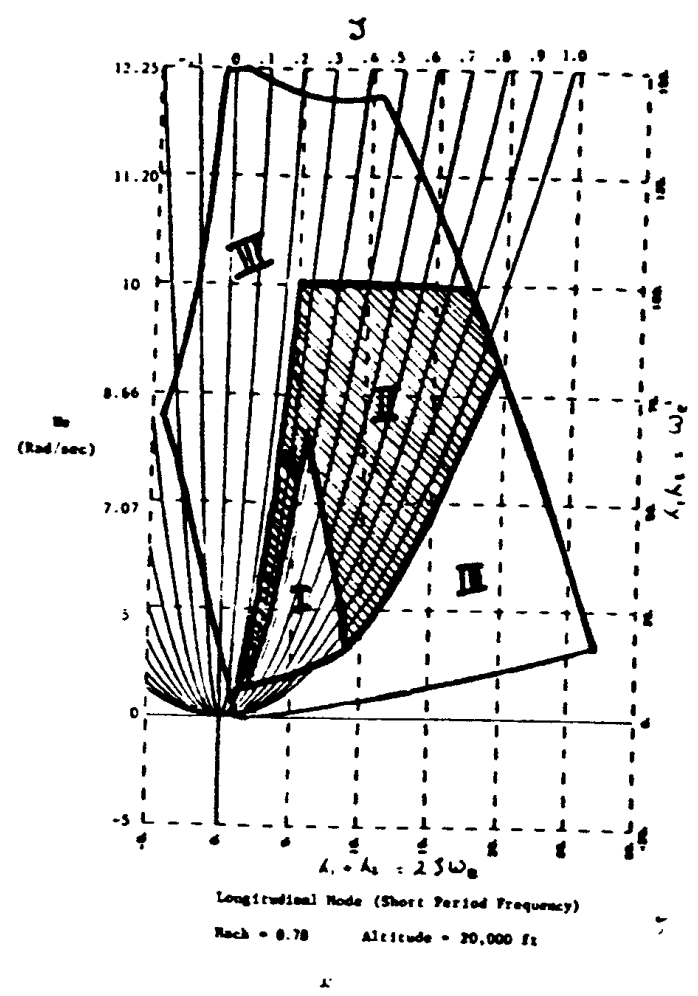

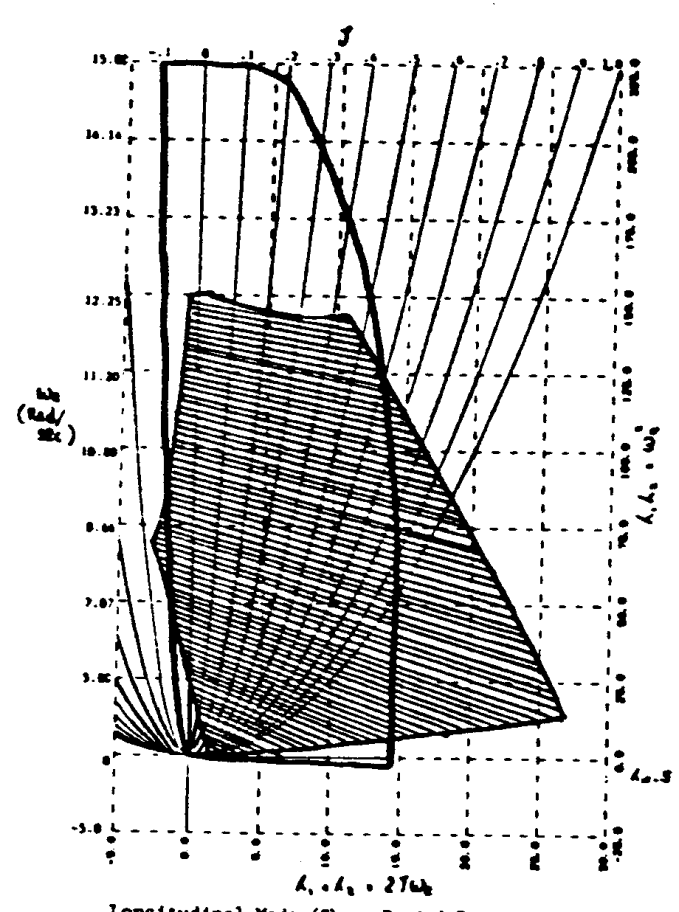

Longleudinal Mode (Shore Period Frequescy)

rach - 0.78 Alicude $=20,000 \mathrm{tz}$

Shaded Area - Analyols Results

Fig. 5 


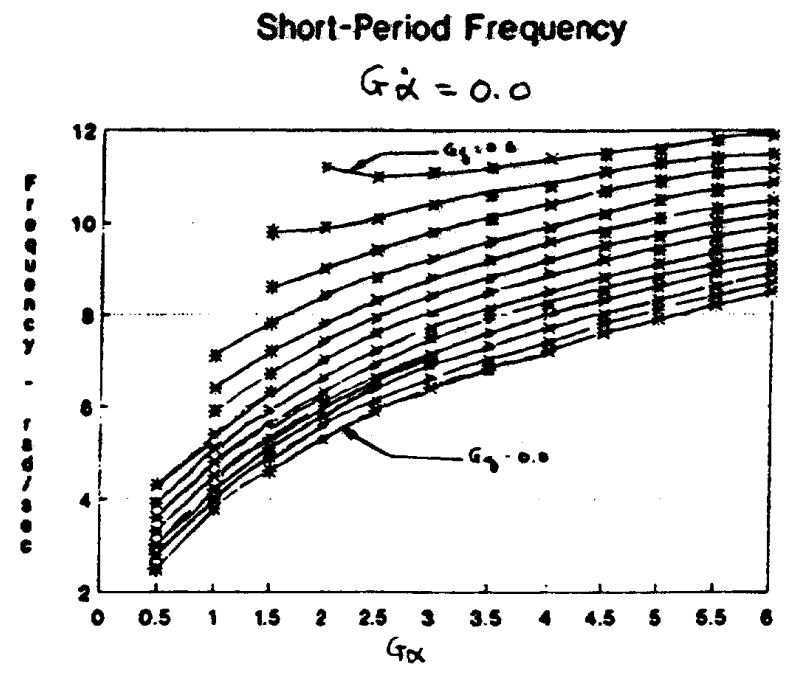

Fig. 6
Time Delay

$\dot{G} \dot{\alpha}=0.0$

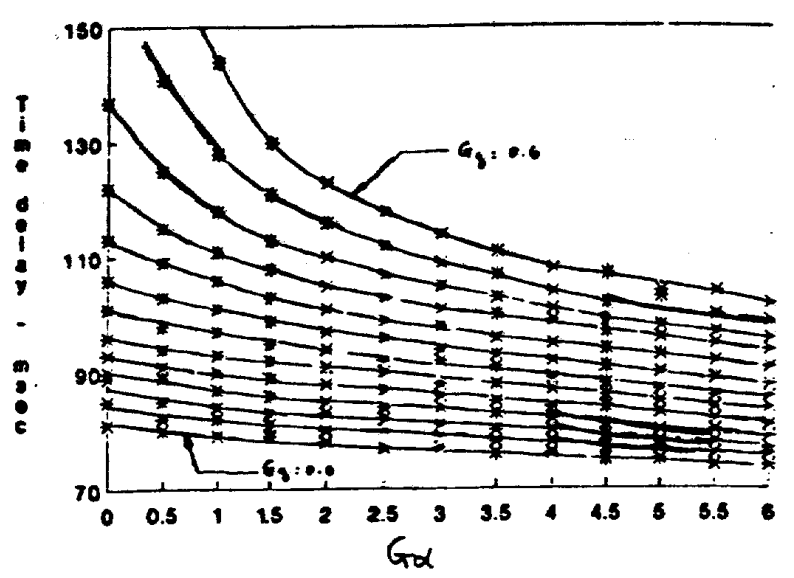

Fig. 8

Short-Period Damping Ratio

$G \dot{\alpha}=0.0$

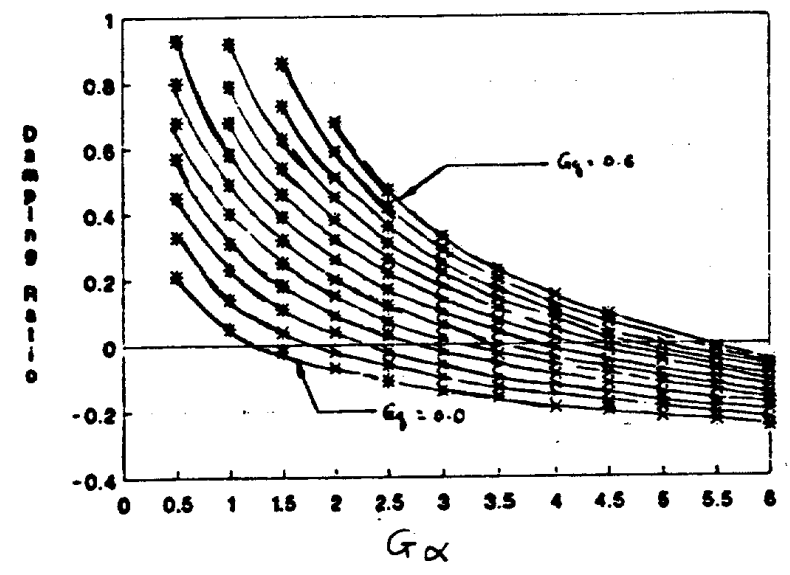

Fig. 7

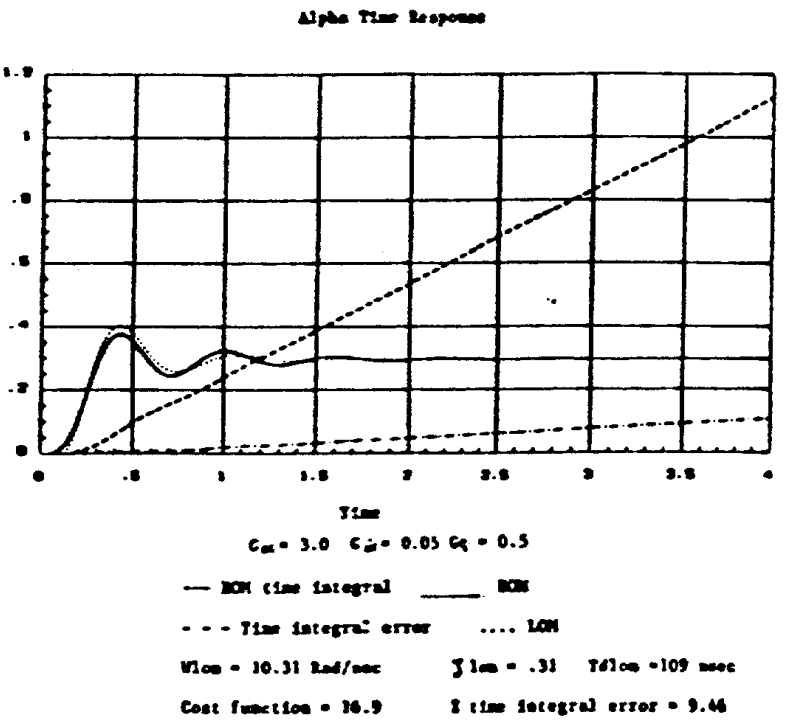

Fig. $19 a$ 


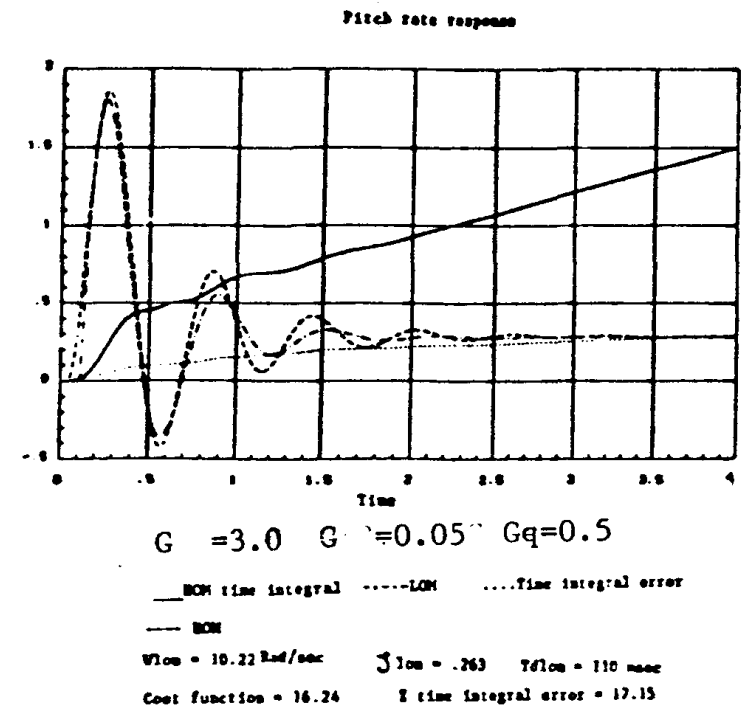

Fig. 9b 